\title{
Operational and environmental evaluation of traffic movement on urban streets using GPS floating-car data
}

\author{
Ahmed Elkafoury ${ }^{1 *}$; Abdelazim Negm ${ }^{1}$, Mohamed Hafez ${ }^{2}$, Mahmoud Bady ${ }^{1}$, Teijiro Ichimura ${ }^{1,3}$ \\ ${ }^{1}$ Environmental Engineering Dept., School of Energy and Environmental Engineering, Egypt-Japan University of Science and \\ Technology (E-JUST), New Borg Al-Arab City Alexandria. Egypt \\ ${ }^{2}$ Transportation Engineering Dept., Faculty of Engineering, University of Alex., Alexandria. Egypt \\ ${ }^{3}$ Department of Chemistry and Materials Science, Tokyo Institute of Technology, Tokyo, Japan \\ * Corresponding author E-mail: ahmed.elkafoury@ejust.edu.eg
}

Copyright (c) 2015 Ahmed Elkafoury et al. This is an open access article distributed under the Creative Commons Attribution License, which permits unrestricted use, distribution, and reproduction in any medium, provided the original work is properly cited.

\begin{abstract}
Evaluating traffic networks is vital for the management of traffic systems. Nowadays, Global Positioning System (GPS) technology, by independent GPS devices or GPS enabled cellular phones, is properly used in most vehicles, especially on urban streets, due to its cost effectiveness, ease of use, and real-time services. With its ability to detect the time position of the floating car, GPS devices introduces a new prospective to gather vehicle information. Collected information can be utilized for traffic management and for Intelligent Transportation System (ITS) to deduce evaluation indicators, and to achieve suitable measures. This paper brings up a framework for using real-time data collected by GPS-floating car technique for evaluating traffic conditions on urban streets. It utilizes GPS data of time, longitude, latitude to estimate evaluation indicators of street segments. This incorporates operational evaluation of street segment by characterizing Level of Service and level of congestion, and incorporates environmental evaluation by estimating the road-side concentration of pollutants emitted by traffic. Framework formation has been described. Different models used within every step of the framework have been investigated. Including models used in GPS data sample analysis, through models used to identify of street segments, and finally models used for street segments evaluation.
\end{abstract}

Keywords: Environmental Evaluation, Floating Car; Global Positioning System (GPS); Level of Service and Level of Congestion; Traffic Conditions on Urban Streets.

\section{Introduction}

Estimating traffic conditions of different vehicular dynamics using real-time traffic information is a challenge for transportation planners. This challenge falls in line with the steady increase in urbanization and in the sequel, the number of vehicles. It has also become the backbone of the Intelligent Transportation Systems (ITS). Conventional traffic data systems utilize fixed sensors to collect real-time traffic information. In fact, traditional fixed detectors; rubber tubes, inductive loops, electric based, video camera and radar have limited amount of sensor infrastructure, and consequently, distribution restrictions [1] because it does not make sense to fix huge number of fixed sensors at every street. Moreover, traditional loop detectors are subjected to errors in measurements. For instance, according to Performance Measurement System in California, 30\% of the traffic detectors are subjected to a daily breakdown [2]. In addition, fixed traffic detector devices can only cover data collection on a limited section of the road facility [3]. More and more, the installation and maintenance costs prevent the deployment of these technologies for the entire arterial network and even for highways in numerous places around the world [4] especially in developing communities.

Nowadays, large numbers of vehicles in major cities are Global Positioning System (GPS) equipped; by independent GPS devices or GPS enabled cellular phones. By which, vehicles act as probes, that produces considerable of high resolution GPS traces. GPS tools used in driver populations are enough to provide accurate measurements of the velocity of the traffic flow [5], and can largely assisted in overcoming the problems associated with conventional traffic data collecting methods [6]. In this concern, a floating car equipped with GPS device can act as a traffic agent on the 
transportation network to collect real-time traffic data. It gives the advance of more accurate, more comprehensive, and larger scale data collecting [7].Furthermore, information about the position and time of the car can be gathered during floating within the traffic flow. It is considered viable source of traffic operating data especially in case of urban road networks.

Collected information is used for the traffic flow analysis. It can be sent periodically to a server. And by using successive GPS samples, the travel time, distance, travel speed, and acceleration can be calculated. This helps infer trends of the operational and environmental characteristics on the road link under investigation through deriving indicators about the operational situation; Level of Service (LOS) and time delay, and environmental situation; emission factors and noise levels. It can also be used for providing vehicle classification information [8]. In addition, GPS data gathered in real-time as well as historical data could be analyzed and processed to be sent back to mobile devices as a tool of traffic control within an Intelligent Transportation System (ITS) and dynamic route guidance for travelers. Moreover, analyzed data can also be sent to a control system to be graphical represented [9]. It also helps in policy planning across a variety of domains including traffic management, urban planning and environmental monitoring [10]. This article proposes a framework for using GPS floating car data samples to evaluate urban street networks. This evolves traffic situation evaluation and environmental evaluation of different street segments. This paper is organized as follows: Section 2 introduces the steps involved in the proposed framework, and the framework structure and formation. Section 3 presents methods, hypothesis and models used in the framework and for the operational and environmental evaluation of the traffic movement on the street segments. Section 4 concludes this work and draws the future research.

\section{Framework formation}

The proposed framework utilizes the GPS samples recorded on the floating car to induces operational and environmental indicators of the urban road network. The GPS device is set on the floating car to record traces every time step according to both needed quality measurement and the GPS device accuracy. Some GPS devices can be set to record time and position samples in a very fine time resolution up to $1 \mathrm{sec}$.

The proposed framework is made up of 3 main successive stages. The first stage concerns GPS data processing, the second evolves the identification of street segments, and the third stage evaluates the street segments. In the first stage, the GPS data traces captured by the GPS device on the floating car are imported to a computer device. This data includes of the longitude, latitude, and time. Then, the data is processed to calculate the ground distance and the travel time between every two successive GPS samples. In the second stages, the street segments is identified and given specific ID. This stage also encloses determination of the actual length of the street segments besides the travel time of the floating car on the segment. The evaluation of the road links is performed in the third stage of the framework. In which the outputs of the street segment identification stages is put to be used for operational and environmental evaluation of different street segments. The framework layout is shown in Figure 1.

\section{Methods and models used in the framework}

This part of the paper describes the models and methods involved in each stage of the framework. It also investigates equations used to express every model.

\subsection{Calculating ground distance between successive data samples}

First step in the framework is to calculate the length (Dm) i.e. ground distance between every two successive data traces. Dm between any two successive position samples ( $n$ and $n+1)$ can be calculated using the points coordinates (latitude and longitude of each). Initially the distance between the 2 successive longitudes $(\mathrm{H})$ and between 2 successive latitudes (V) are calculated as follows:

$\mathrm{H}=69.1\left(\operatorname{Lon}_{\mathrm{n}+1}-\operatorname{Lon}_{\mathrm{n}}\right)$

$\mathrm{V}=69.1\left(\mathrm{Lat}_{\mathrm{n}+1}-\mathrm{Lat}_{\mathrm{n}}\right)$

$\mathrm{D}_{\mathrm{m}}=\sqrt{\mathrm{H}^{2}+\mathrm{V}^{2}}$

Where $\operatorname{Lon}_{n}$ and $\operatorname{Lon}_{n+1}$ are the longitude of the $n$ and the $n+1$ point respectively. Lat $n$ and Lat $n+1$ are the latitude of the $n$ and the $n+1$ point respectively. Since the unit difference between two successive longitudes or two successive longitudes is 69.1 mile, a factor of 69.1 is used in for conversion.

As a matter of fact, equations used for calculating $(\mathrm{H})$ and $(\mathrm{V})$ in this framework is not providing high accuracy of calculating (Dm) since it neglects the earth surface curvature. But, this accuracy is sufficient in urban areas since the road network in urban cities is in areas with maximum radius of about $30 \mathrm{~km}$. In such cases, the effect of earth's curvature on measurement is so small that we can hardly measure it. So, no significant difference between the curved distance and corresponding straight distance and the curvature of the earth surface can be neglected. In other words, horizontal lines are assumed to be straight line and all vertical lines are parallel [11]. 


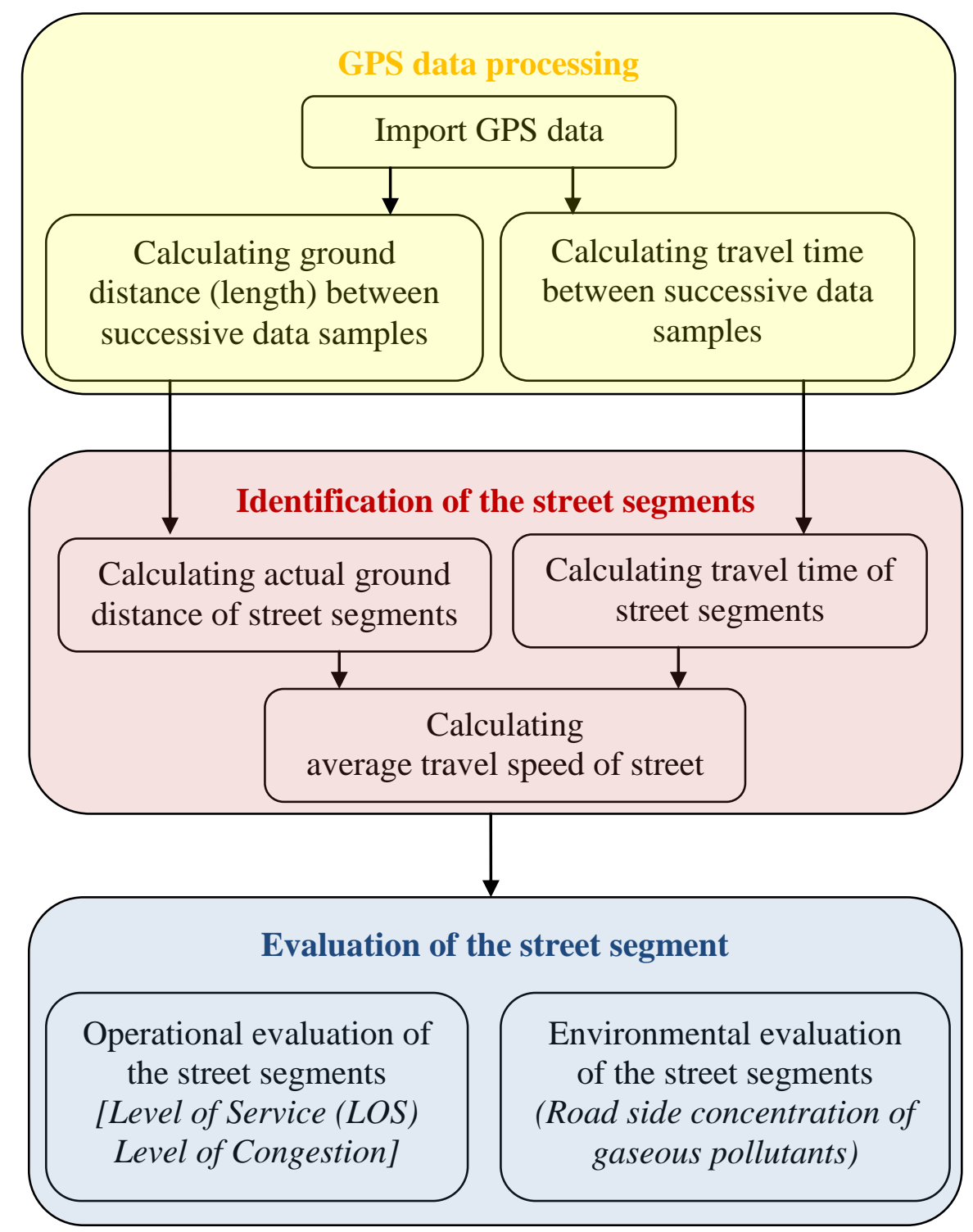

Fig. 1: Stages Constituting Framework for the Operational and Environmental Evaluation of Urban Streets Based on GPS Data

\subsection{Calculating travel time between successive data samples}

Second stage in the proposed framework evolves the determination of the travel time between every two successive data traces. If the registered times at two successive GPS samples are trn and trn+1, then the travel time (tm) is: $\mathrm{t}_{\mathrm{m}}=\left(\mathrm{tr}_{\mathrm{n}+1}-\operatorname{tr}_{\mathrm{n}}\right)$

The dimensions of (tm) depend on the dimensions of the registered time on the GPS data samples. To convert dimensions of (tm) to any other required dimensions, it can be multiplied by the suitable conversion factor.

\subsection{Calculating actual ground distance of street segments}

The segmentation process is performed on the bases of the preferred segment length based on the required level of analysis and the desired accuracy. Which means that, defining a specific street segment length is the transportation planner decision. In this framework, the identification of the street segments is done in the third stage. In which an ID number is assigned to each segment to refer the operational and environmental indicators to this ID when they are calculated.

After deciding the street segment length (L), the exact length of the street segment (Le) is determined with an accuracy of ( \pm average of Dm), i.e.:

$\mathrm{L}_{\mathrm{e}}=\sum\left(\mathrm{D}_{\mathrm{m} 1}+\mathrm{D}_{\mathrm{m} 2}+\mathrm{D}_{\mathrm{m} 3}+\ldots \ldots+\mathrm{D}_{\mathrm{mN}}\right)$

Where Dm1 is the ground distance between the first and second GPS samples, Dm2 is ground distance between the second and third GPS samples, and DmN is ground distance between the Nth and (N+1)th GPS. N is the number of ground distances composing the street segment. Which satisfies the condition that Le $=\mathrm{L} \pm$ (average of Dm). This 
means that if the street segment consists of $\mathrm{N}$ ground distances, then the street segment includes $\mathrm{N}+1$ points i.e. $\mathrm{N}+1$ GPS data samples. Finally, when the segment is defied, it is given an ID number that will be used in further framework steps.

\subsection{Calculating travel time of street segments}

The forth step of the framework represents estimating the total travel time $(\mathrm{T})$ of the street segment. It can be calculating by summing the travel time $(\mathrm{tm})$ of the $(\mathrm{N})$ ground distances composing the segment as illustrated in the following formula:

$\mathrm{T}=\sum_{\mathrm{i}=1}^{\mathrm{N}} \mathrm{t}_{\mathrm{m}}$

\subsection{Calculating average travel speed of street segments}

Total travel time (T) is used as input for the fifth stage of the framework to calculate the average travel speed of the street segment. It is one of the most important indexes for traffic status identification [Average travel speed estimation]. In the fifth stage of the proposed framework, the average travel speeds (V) of the floating car over the street segments can be estimated in by dividing the exact length of the segment (Le) by the travel time (T) of the street segment as follows:

$\mathrm{V}=\frac{\mathrm{L}_{\mathrm{e}}}{\mathrm{T}}$

The calculated average travel speeds of each segment should be assigned to the segment's ID. Here, it is important to mention that the dimensions of (V) depend on the dimensions of calculated (Le) and (T).

\subsection{Operational evaluation of street segments}

The aim of the operational evaluation of any street segment is to identify the road state depending on different measures of effectiveness. Traffic density, average travel speed, average travel time, level of service, level of congestion can be used to describe the operational conditions of the current state of the road link. In this paper, two parameters are considered for the operational evaluation of different street segments. The first is the Level of Service (LOS), and the second is the level of congestion.

\subsubsection{Level of service}

Level of Service (LOS) is a quantitative measure of the performance and quality of the traffic service. It is the used indicator in all Highway Capacity Manuals (HCM) versions since 1965 to 2010 versions. On a scale from (A) to (F), the traffic facility is assigned to one of six levels of services, with LOS (A) representing the best operating conditions and LOS (F) the worst. To define LOS, different Measures of Effectiveness (MOE) have been considered in the HCM series including travel time, traffic flow to capacity ratio, traffic density, and travel speed.

In this framework, the operational evaluation of the urban street segment is done using the linear function mentioned in Multimodal level of service analysis of urban streets report 616 of NCHRP [12]. This function utilizes the average travel speed of the street segment and the urban street class to calculate the LOS as follows:

LOS $=$ Integer $\{(0.151231 * \mathrm{~V})+(0.636927 *$ Class $)-2.17765\}$

Where LOS is the HCM LOS integer scale (where $A=5, B=4, C=3, D=2, E=1, F=0$ ), Integer is the integer function (rounds off the value to the nearest integer value), (V) is the average travel speed of through traffic on the street segment (mph), and Class is the arterial class as classified by HCM as shown in Table 1.

\subsubsection{Level of congestion}

In cities, traffic congestion is a hot issue that affects the urban population lives. It can be stated as the reduction of traffic speeds accompanied by losing the ability to maneuver resulting from intensive traffic movements on road network links. The cardinal rule for alleviating traffic congestion is to scientifically and quantitatively describe traffic congestion. In the proposed framework, to detect the traffic congestion levels on the street segments; the normalized Traffic Congestion Index (TCI) [14] is selected as indicator of the traffic congestion level. In which the speed of the

Table 1: Classification of Urban Streets According to HCM [13]

\begin{tabular}{llll}
\hline \multirow{2}{*}{ Default Free Flow Speed $(\mathrm{km} / \mathrm{h})$} & \multirow{2}{*}{ Design Category } & Classification \\
\cline { 3 - 4 } & & Principal Arterial & Minor Arterial \\
\hline 80 & Suburban & I & N/A \\
65 & Intermediate & II & II \\
55 & Urban & III or IV & II or IV \\
45 & IV \\
\hline
\end{tabular}


street segment is normalized to the design speed of the road $(\mathrm{Vd})$ in an exponential function model to quantify the travelers' perception about traffic performance as follows:

$\mathrm{TCI}=\left[12.96\left(\frac{\mathrm{V}}{\mathrm{v}_{\mathrm{d}}}\right)\right]-\left[2.96\left(\frac{\mathrm{V}}{\mathrm{v}_{\mathrm{d}}}\right)^{2}\right]$

If the design speed (VD) of the urban arterial is not known, it can be assumed to be equal to the typical free flow speed of the arterial category as in Table 1. The travelers' perception about traffic movement performance can be indicated from the TCI level on a normalized scale from 0 to 10.In which, higher values of TCI level indicate smooth traffic movement and vice versa. The specific definition of each level is shown in Table 2.

\subsection{Environmental evaluation of street segments}

Unfortunately, transport is contributing highly to a number of environmental problems including noise, different kinds of emissions, besides climate change. Estimations expect the transportation sector to be the most rapidly growing source of $\mathrm{CO} 2$ emission over the next 30 years, increasing at an annual rate of $2 \%$ to $3 \%$. From which, developing countries contribute of about $63 \%$ of GHG emissions by the year 2030 [15]. Recently, the importance of sustainable oriented planning of urban transportation systems has been a focus concern specially for assessing the urban air quality. This raises the need to evaluate not only the traffic performance of road network link, but also its environmental impact. In this concern, transportation emission models are part of the modeling process of transportation planning. It introduces an important tool for the environmental evaluation of different transportation scenarios [16]. In this paper, the environmental evaluation of the street segment is done based on road-side concentration model of six gaseous pollutants emitted by vehicles; CO, SO2, NO2, NO, PM10 and PM2.5.

The first step to estimate the road-side pollutants concentration is to determine the traffic volume of different vehicle type; passenger car, bus, truck, lorry and motorcycle. It can be taken as the product of multiplying the percentage of the vehicle type in the traffic composition of the study area, by the traffic flow on the street segment (F). For example:

$\mathrm{F}_{\mathrm{P}}=\%$ Passenger car in traffic composition $* \mathrm{~F}$

To estimate the traffic flow on the street segment under investigation, the classic (Bureau of Public Roads) BPR equation [urban arterial] which predict the average travel speed as a function of the traffic flow (F) (veh/hr) to capacity ratio $(\mathrm{Ca})(\mathrm{veh} / \mathrm{hr})$. The equation takes the following form:

$\mathrm{V}=\frac{\text { free flow speed }}{1+0.15\left(\frac{\mathrm{F}}{\mathrm{Ca}}\right)^{4}}$

From which the traffic flow $(\mathrm{F})$ can be expressed as:

$\mathrm{F}=\mathrm{Ca}\left[\sqrt[4]{\frac{\left(\frac{\text { free flow speed }}{\mathrm{V}}-1\right)}{0.15}}\right]$

Regarding the capacity of the street segment, it is estimated using the HCM methodology which proposes a capacity of $1800 \mathrm{veh} / \mathrm{hr} / \mathrm{lane}$ of the urban street facilities [13].

In the second step to estimate the road-side concentration of pollutants, the framework utilizes the equation describes the relations between the road side gaseous concentration and average link speeds [17] is utilized. This equation can be applied to different vehicle types on urban streets, and considers the traffic composition of the study area. The equations have the following exponential structure:

$\mathrm{C}_{\mathrm{i}}=\mathrm{F}_{\mathrm{P}} \alpha_{\mathrm{iP}} \mathrm{V}^{\beta_{\mathrm{iP}}}+\mathrm{F}_{\mathrm{b}} \alpha_{\mathrm{ib}} \mathrm{V}^{\beta_{\mathrm{ib}}}+\mathrm{F}_{\mathrm{t}} \alpha_{\mathrm{it}} \mathrm{V}^{\beta_{\mathrm{it}}}+\mathrm{F}_{\mathrm{l}} \alpha_{\mathrm{il}} \mathrm{V}^{\beta_{\mathrm{il}}}+\mathrm{F}_{\mathrm{m}} \alpha_{\mathrm{im}} \mathrm{V}^{\beta_{\mathrm{im}}}$

where $\mathrm{Ci}$ is the road side concentration of the gaseous pollutant $\mathrm{i}(\mathrm{CO}, \mathrm{SO} 2, \mathrm{NO} 2, \mathrm{NO}, \mathrm{PM} 10$ and PM2.5) (mg/m3), (V) is the average travel speed of through traffic on the street segment $(\mathrm{km} / \mathrm{hr})$, and $(\alpha)$ and $(\beta)$ are equation constants for different pollutants according to vehicles types as shown in Table 3. (F) Is the traffic volume of a specific vehicle type ( $\mathrm{p}$ is passenger car, $\mathrm{b}$ is bus, $\mathrm{t}$ is truck, $\mathrm{l}$ is lorry and $\mathrm{m}$ is motorcycle).

Table 2: TCI Categories According to Quantifications of Travelers' Perception about Traffic Performance Categories [14].

\begin{tabular}{llllll}
\hline $\begin{array}{l}\text { Perception about Traffic } \\
\text { Performance }\end{array}$ & Very smooth & Smooth & Relatively smooth & Moderately congested & $\begin{array}{l}\text { Heavily } \\
\text { congested }\end{array}$ \\
\hline TCI & 8 to 10 & 6 to 8 & 4 to 6 & 2 to 4 & 0 to 2 \\
\hline
\end{tabular}

Table 3: (A) and (B) Values for the Concentration Equation of Roadside Pollutants from Various Vehicle Types [17].

\begin{tabular}{|c|c|c|c|c|c|c|c|}
\hline Pollutant & Vehicle type & $\alpha$ & $\beta$ & Pollutant & Vehicle type & $\alpha$ & $\beta$ \\
\hline \multirow{5}{*}{$\mathrm{CO}$} & Passenger car & 14.228 & -0.423 & \multirow{5}{*}{$\mathrm{NO}$} & Passenger car & 2.029 & -0.616 \\
\hline & Bus & 18.304 & -0.536 & & Bus & 28.496 & -0.374 \\
\hline & Truck & 34.046 & -0.665 & & Truck & 112.410 & -0.978 \\
\hline & Lorry & 17.52 & -0.610 & & Lorry & 42.212 & -0.665 \\
\hline & Motorcycle & 9.195 & -0.527 & & Motorcycle & 1.188 & -0.358 \\
\hline
\end{tabular}




\begin{tabular}{llllllll}
\hline & Passenger car & 0.105 & -0.608 & & Passenger car & 0.025 & -0.580 \\
$\mathrm{SO}_{2}$ & Bus & 0.090 & -0.413 & & Bus & 1.635 & -0.664 \\
& Truck & 0.256 & -0.601 & $\mathrm{PM}_{10}$ & Truck & 2.512 & -0.657 \\
& Lorry & 0.191 & -0.357 & & Lorry & 3.469 & -0.591 \\
& Motorcycle & 0.041 & -0.384 & & Motorcycle & 0.071 & -0.364 \\
\hline \multirow{3}{*}{$\mathrm{NO}_{2}$} & Passenger car & 0.394 & -0.616 & & Passenger car & 0.009 & -0.461 \\
& Bus & 3.498 & -0.407 & & Bus & 0.940 & -0.681 \\
& Truck & 11.109 & -0.956 & $\mathrm{PM}_{2.5}$ & Truck & 1.354 & -0.657 \\
& Lorry & 4.838 & -0.659 & & Lorry & 1.714 & -0.567 \\
& Motorcycle & 0.067 & -0.317 & & Motorcycle & 0.024 & -0.193 \\
\hline
\end{tabular}

\subsection{Conclusion and recommendations}

This paper introduced a proposed framework to use real-time GPS data collected by a floating car for the purpose of operational and environmental evaluation of the traffic situation on urban street segments. This framework is useful for traffic management of urban streets. It might be incorporated as a part in an Intelligent Transportation System (ITS) to increase to performance of the involved ITS. Different models and equations used within the framework process have been investigated and explained. Results showed that it is possible to use the position and time GPS data collected by the floating car to infer the travel time, segment lengths, and average travel speed of the street segment. From which, the traffic situation can be evaluated by estimating the LOS, level of congestion, and also the road-side concentration of different pollutants emitted by vehicles in traffic.

As future work, it is intended to validate the presented framework by applying it to a case study. The proposed framework might be extended to include additional operational and environmental indicators such as roadside noise levels, and delay time over street segments.

\section{Acknowledgements}

The first author would like to thank Egyptian Ministry of Higher Education (MOHE) for providing him the financial support (PhD scholarship) for this research as well as the Egypt Japan University of Science and Technology (E-JUST) for offering the facility and tools needed to conduct this work.

\section{References}

[1] Zhou Xiangyu, Wenjun Wang, and Long Yu. "Traffic Flow Analysis and Prediction Based on GPS Data of Floating Cars." Proceedings of the International Conference on Information Technology and Software Engineering. Springer Berlin Heidelberg 2013.

[2] Bar-Gera, H. "Evaluation of a cellular phone-based system for measurements of traffic speeds and travel times: a case study from Israel", Transportation Research C 15 (6), 380-391., 2007. http://dx.doi.org/10.1016/j.trc.2007.06.003.

[3] Xiaohui, S., Jianping, X., Jun, Z., Lei, Z., \& Weiye, L., "Application of dynamic traffic flow map by using real time GPS data equipped vehicles". In ITS Telecommunications Proceedings, 6th International Conference on (pp. 1191-1194). IEEE. 2006.

[4] Herrera, Juan C., Work, D. B., Herring, R., Ban, X. J., Jacobson, Q., \& Bayen, A. M., "Evaluation of traffic data obtained via GPS-enabled mobile phones: The $<$ i $>$ Mobile Century</i $>$ field experiment." Transportation Research Part C: Emerging Technologies 18.4 (2010): 568 583. http://dx.doi.org/10.1016/j.trc.2009.10.006.

[5] W.-H. Lee, S.-S. Tseng, S.-H. Tsai, "knowledge based real-time travel time prediction system for urban network, Expert Systems with Applications". ELSEVIER 36 (2009) 4239-4247. http://dx.doi.org/10.1016/j.eswa.2008.03.018.

[6] Byon, Y-J., Amer Shalaby, and Baher Abdulhai. "Travel time collection and traffic monitoring via GPS technologies." Intelligent Transportation Systems Conference, 2006. ITSC'06. IEEE. IEEE, 2006.

[7] Yong-chuan, Zhang, Zuo Xiao-qing, and Chen Zhen-ting. "Traffic Congestion Detection Based On GPS Floating-Car Data." Procedia Engineering 15 (2011): 5541-5546. http://dx.doi.org/10.1016/j.proeng.2011.08.1028.

[8] Sun, Zhanbo, and Xuegang Jeff Ban., "Vehicle classification using GPS data." Transportation Research Part C: Emerging Technologies 37 (2013): 102-117. http://dx.doi.org/10.1016/j.trc.2013.09.015.

[9] Jiménez-Meza, A., J. Arámburo-Lizárraga, and E. de la Fuente, "Framework for Estimating Travel Time, Distance, Speed, and Street Segment Level of Service (LOS), based on GPS Data", Procedia Technology 7 (2013): 61-70. http://dx.doi.org/10.1016/j.protcy.2013.04.008.

[10] Pang, L. X., Chawla, S., Liu, W., \& Zheng, Y., "On detection of emerging anomalous traffic patterns use GPS data", Data \& Knowledge Engineering 87 (2013): 357-373. http://dx.doi.org/10.1016/j.datak.2013.05.002.

[11] Wuttiet Tafesse and Tesfaye Gobena, Lecture Notes for Environmental Health Science Students - Surveying, Haramaya University, 2005.

[12] Dowling, Richard G., et al., "NCHRP Report 616: multimodal level of service analysis for urban streets", Transportation Research Board of the National Academies, Washington, DC (2008).

[13] Transportation Rresearch Board, Highway capacity manual, National Research Council (2000).

[14] Liu, M., Yu, L., Guo, J., Guo, S., Guo, J., \& Wen, H. "Fuzzy logic-based urban traffic congestion evaluation models and application", Proc., 1st Int. Conf. on Transportation Engineering. New York: ASCE, 2007.

[15] Gumusay, M. Umit, Alper Unal, and Rukiye Aydın., "Use of geographical information systems in analyzing vehicle emissions: Istanbul as a case study", The International Archives of the Photogrammetry, Remote Sensing and Spatial Information Sciences 37 (2008): 997-1000.

[16] Elkafoury, A., Negm, A. M., Bady, M., \& Aly, M. H. F., "Review of transport emission modeling and monitoring in urban areas-Challenge for developing countries", Advanced Logistics and Transport (ICALT), 2014 International Conference on. IEEE, 2014.

[17] Pu, Yichao, and Chao Yang., "Estimating urban roadside emissions with an atmospheric dispersion model based on in-field measurements", Environmental Pollution 192 (2014): 300-307. http://dx.doi.org/10.1016/j.envpol.2014.05.019. 